\title{
Online Pyrometry for Weld Seam Localization in Absorber-Free Laser Transmission Welding of Transparent Polymers
}

\author{
Frederik Maiwald*, Stephan Englmaier and Stefan Hierl \\ Ostbayerische Technische Hochschule Regensburg, Labor Lasermaterialbearbeitung, \\ Technologie Campus Parsberg-Lupburg, Am Campus 1, 92331 Parsberg, Germany \\ *Corresponding author's e-mail: frederik.maiwald@oth-regensburg.de
}

\begin{abstract}
Production of optical and medical devices makes high demands on cleanliness, precision, visual appearance and reliability. Advantages of laser transmission welding - contactless input of energy, high precision, no adhesives and no particle formation - can be fully exploited for this use. For absorber-free welding, lasers emitting in the polymers' intrinsic absorption spectrum between $1.6 \mu \mathrm{m}$ and $2 \mu \mathrm{m}$ are used. Focusing the laser beam with high NA provides large intensity gradients inside the specimen and enables selective fusing of the joining zone without affecting the surface. Since a molten upper surface leads to visible and palpable irregularities and causes warpage, the vertical expansion of the weld seam is crucial. To meet the high quality and reliability required in the medical and optical industry, online monitoring of the seam is necessary. The aim of this work is the localization and evaluation of the weld seam using pyrometry. First, the emitted thermal radiation is computed, enabling signal analysis regarding origin and spectrum. Thus, assessment of measurement spectrum and forecast of the signal in dependence on seam shape and position is possible. Finally, welding tests with Topas $8007-04$ are performed at $200 \mathrm{~mm} / \mathrm{s}$ feed rate and monitored with $100 \mathrm{kHz}$. The comparison of tracked data with thin-cuts demonstrates that weld seam localization by pyrometry is possible, enabling online assessment of the process.
\end{abstract}

DOI: $10.2961 /$ jlmn.2021.01.2002

Keywords: laser transmission welding, pyrometry, absorber-free, transparent polymers, process monitoring, thermal radiation

\section{Introduction}

Manufacturing of optical and medical devices makes high demands on cleanliness, precision, visual appearance and reliability. Advantages of absorber-free laser transmission welding - contactless input of energy, high precision, no adhesives and no particle formation - can be fully exploited for this application.

Figure 1 (a) shows the process principle of absorber-free laser transmission welding. Both approx. $1 \mathrm{~mm}$ thick joining partners are clamped in overlap. A laser beam with wavelength in the polymers' intrinsic absorption spectrum between $1.6 \mu \mathrm{m}$ and $2 \mu \mathrm{m}$ is focused into the joining zone. Focusing the beam with high NA provides large intensity gradients inside the specimen, enabling selective fusing of the joining zone. Thus, processing of hermetically sealed seams with a size of only a few tenths of a millimeter is possible without affecting the surface of the upper joining partner. Especially the vertical expansion of the seam is crucial for welding, since a molten surface leads to visible and palpable irregularities, whereas a too short seam causes instability and inadequate tightness [1-3].

To fulfill the extreme demands on quality and productivity in the medical industry, online process monitoring is necessary. In contrast to post process evaluation, online monitoring enables closed loop control and does not require additional, time-consuming test cycles.

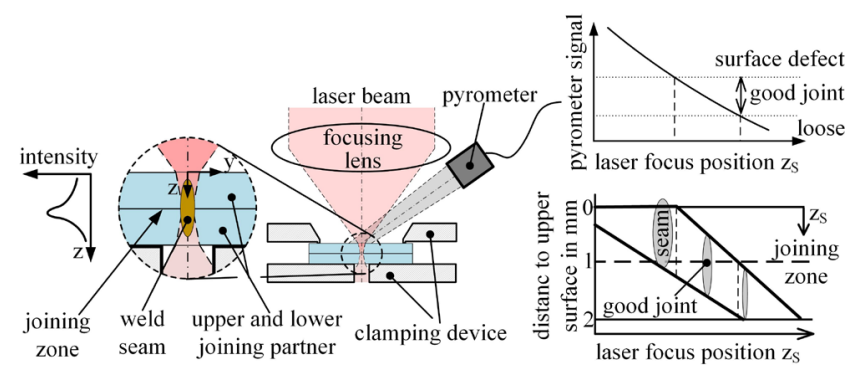

(a)

(b)

Fig. 1 (a) Processing setup with high NA focusing optic and offaxis pyrometer; (b) Sketch of the pyrometer signal for weld seam evaluation.

In transparent-absorbent laser polymer welding, pyrometry is already established for online temperature measurement with several tens of $\mathrm{kHz}$ [4-7]. Since the lower partner is blackened here, the joining zone acts as a surface emitter [7]. In contrast, the radiation source in absorber-free welding of transparent polymers is not a distinct surface but a three-dimensional volumetric emitter.

Similar to other semi-transparent materials, such as glass, the received radiation will arise not only from the upper surface but also from the interior of the specimen. Within the material, radiation is being generated and absorbed simultaneously. The radiation generated internally must travel to the surface before being emitted and is attenuated on this path according to Lambert's law $[8,9]$. To be able to make distinct conclusions on the process' temperature course, the interplay of emission and re-absorption inside the 
material must be known. Additionally challenging, the pyrometer converts all received radiation emitted by the three dimensional interior only to a scalar value.

The goal of this work is to identify a pyrometer enabling detection of both surface defects and loose joints (see figure $1 \mathrm{~b}$ ). Thus, the thermal radiation is modeled based on a thermal simulation of the welding process, enabling analysis of the emission regarding wavelength spectrum and depth of origin in dependence on the material and process parameters. To choose the pyrometer's spectral range, the signal is evaluated depending on the laser's focus position, which has a key impact on the crucial vertical expansion of the seam. Subsequently, a fixed-focus optic with an off-axis pyrometer is set up to prove the capability of weld seam localization and evaluation for the cyclic olefin copolymer (COC) Topas 8007-04.

\section{Simulation setup}

To be able to calculate the emitted thermal radiation, a process simulation is set up to compute the temporal and spatial temperature course during welding.

\subsection{Process Model}

In absorber-free laser transmission welding, deformations and melt blowout are usually avoided to prevent a blockage of the microfluidic system. Hence, a thermal finite element analysis neglecting deformations is sufficient. Since the temperature gradient in feed direction is insignificant because of the polymer's low thermal conductivity and feed rates above $100 \mathrm{~mm} / \mathrm{s}$, a twodimensional model is used. The thermal simulation is split into two sections: Calculation of heat energy $Q_{(y, z)}$ deposited by the laser beam and the subsequent computation of the temperature distribution $T_{(y, z, t)}$.

The energy per unit length $Q_{(y, z)}$, deposited during one passage of the laser beam across an 2D intersection of the specimen, is calculated according to equation (1) by integrating the volumetric heat rate $\dot{Q}_{(x, y, z, t)}$ over the time t (in feed direction $\mathrm{x}$ ) in MATLAB [10]. The volumetric heat rate $\dot{Q}$ within the integral equals the three dimensional radiant power of a Gaussian beam absorbed in media [11]. In this formula, $P$ is the laser power, $\alpha$ the material's absorption coefficient and $v_{x}$ the feed rate. The beam diameter $d_{(z)}$ along the optical axis is given by equation (2) depending on the Rayleigh length $z_{R}$, the focal diameter $d_{0}$ and the laser focus position $z_{s}$.

Subsequently, the integrated energy per unit length $Q_{(y, z)}$ is applied to a 2D thermal simulation model and the temporal and spatial temperature course $T_{(y, z, t)}$ after the laser-material-interaction is computed. The initial temperature $T_{(y, z, 0)}$ of the simulation is $23{ }^{\circ} \mathrm{C}$. Heat flow during the passage of the laser and heat transfer to ambient air are neglected. The density of the studied material Topas $8007-04$ is $1.00 \mathrm{~g} / \mathrm{cm}^{2}$ at room temperature. The thermal material properties thermal conductivity $\left(0.15 \mathrm{~W} /\left(\mathrm{m}^{*} \mathrm{~K}\right)\right.$ at $\left.25^{\circ} \mathrm{C}\right)$ and heat capacity $\left(1.34 \mathrm{~J} /\left(\mathrm{g}^{*} \mathrm{~K}\right)\right.$ at $\left.25^{\circ} \mathrm{C}\right)$ are implemented in dependence on temperature. The reflectivity is $7.9 \%$ at $1940 \mathrm{~nm}$ wavelength.

The simulation procedure has already been used successfully for process layout in transparent-absorbent [7,
$10,12]$ and transparent-transparent welding, where it was compared with experimental results for semi-crystalline polyamide 6 [13].

$$
\begin{aligned}
& Q_{(y, z)}=\int_{-\infty}^{+\infty} \frac{8 \cdot \alpha \cdot P}{\pi \cdot d_{(z)}^{2}} \cdot \exp \left(-\alpha \cdot z-8 \frac{\left(v_{x} \cdot t\right)^{2}+y^{2}}{d_{(z)}^{2}}\right) d t \\
& d_{(z)}=d_{0} \sqrt{1+\left(\frac{z-z_{s}}{z_{R}}\right)^{2}}
\end{aligned}
$$

Figure 2 (b) shows the comparison of a welded specimen's thin cut with the computed temperature field directly $(25 \mu \mathrm{s})$ after laser-material interaction. The cut on the right side of figure 2 (b) was prepared using a rotational microtome and photographed in polarized light in a transmitted light microscope. Since COC is an amorphous polymer and no melting of crystallites occurs, analysis of thin-cuts is imprecise compared to semi-crystalline materials. The change of polarization visible in transmitted light at COC is partially caused by the relaxation of stresses and does not necessarily require an excess of melting temperature. Thus, two temperature ranges are marked:

In the greyed region in figure $2(\mathrm{a})$, the melting $T_{\text {melt }}$ temperature of approx. $200{ }^{\circ} \mathrm{C}$ is exceeded and welding of the upper(UP) and lower (LP) joining partner is assumed [14]. The black elliptic line in the 2D-view indicates the isotherm $T_{v i s}$ of $150^{\circ} \mathrm{C}$ and confines the visible region of the seam (see figure $2 \mathrm{~b}$ ). This temperature is determined by comparing experiment and simulation. To achieve a good seam - ensuring bonding without affecting the upper surface - the joining zone must exceed $T_{\text {melt }}$ and the surface must not exceed $T_{v i s}$

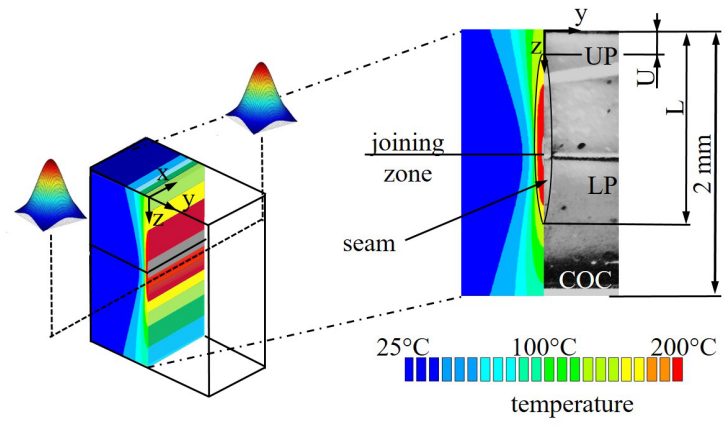

(a)

(b)

Fig. 2 (a) Computed temperature field $25 \mu$ s after laser-material interaction: feed rate $=200 \mathrm{~mm} / \mathrm{s}$, laser power $=60 \mathrm{~W}$, laser focus position $z_{S}=1.1 \mathrm{~mm}$, Rayleigh length $=0.3$; (b) Comparison of temperature field and related weld seam in cross sections. Distance between specimen's surface and upper (U) and lower (L) end of the weld seam. The upper (UP) and lower (LP) joining partner are each $1 \mathrm{~mm}$ thick and made of the same material Topas 8007-04.

\subsection{Modelling detectable radiation}

Pyrometers spatially average the received radiation emitted by the specimen's volume and only return a scalar value. To be able to draw conclusions, the signal's spectrum and place of origin must be known in dependence on the process and material parameters. Therefore, the radiation emitted from the interior of the specimen is modelled in MATLAB, considering the interplay of emission and reabsorption, whereas reflection at the surface is neglected. For calculation, the pyrometers optical axis is aligned with 
the $\mathrm{z}$-axis. Since the computation is simplified to one dimension along the optical axis, solid angle and detection spot area are not taken into account.

The radiation $L_{S(\lambda)}$ - per area and solid angle - detectable at the upper surface of a specimen with thickness $s$ is calculated based on the given temperature course considering blackbody emission $L_{B(\lambda, T)}$, emissivity $\epsilon_{(\lambda)}$ and transmissivity $\tau_{(\lambda, z)}$ according to equation (3) [7, 15]. Equation (4) describes the spectral emission of a black body $L_{B(\lambda, T)}$ according to Planck's law in dependence on the temperature $T$. Speed of light $c$, Planck's constant $h$ and Boltzmann constant $k$ are constant parameters. Equation (5) gives the emissivity $\epsilon_{(\lambda)}$ of a semi-transparent increment with thickness $d z$ and coefficient of absorption $\alpha$. The absorption is calculated by Lambert Beer's law, which is also used to calculate the attenuation of radiation emitted inside the specimen. The transmittance $\tau_{(\lambda, z)}$ of radiation, initially emitted by an increment with distance $z$ from the surface, is given by equation (6).

$$
\begin{aligned}
& L_{S_{(\lambda)}}=\int_{0}^{S} L_{B(\lambda, T)} \cdot \epsilon_{(\lambda)} \cdot \tau_{(\lambda, z)} d z \\
& L_{B(\lambda, T)}=\frac{2 h c^{2}}{\lambda^{5}} \frac{1}{e^{\left(\frac{h c}{\lambda k T}\right)}-1} \\
& \epsilon_{(\lambda)}=1-e^{-\alpha_{(\lambda)} * d z} \\
& \tau_{(\lambda, z)}=e^{-\alpha_{(\lambda)} * z}
\end{aligned}
$$

The pyrometer's electric signal is computed by multiplying the detectable radiation $M_{S_{(\lambda)}}$ with the pyrometer's spectral sensitivity $I_{(\lambda)}$ and integrating over the wavelength spectrum.

\section{Signal Analysis}

To be able to choose the best measurement spectrum, the fundamental correlation of absorption, wavelength and radiation origin is analyzed. Subsequently, the signal of different available pyrometer chips is computed and evaluated regarding weld seam localization.

\subsection{Spectral and Spatial Composition}

Figure 3 (a) shows the cross section of an exemplary temperature field $T_{(y, z, 25 \mu s)}$ right after laser-material interaction, enabling tight weld seams without surface defects. Section (b) shows the spectrally resolved radiation $L_{S_{(\lambda, \alpha)}}$ detectable at the specimen's surface according to equation (3). The radiation is integrated along the $z$-axis at $\mathrm{y}=0$. Section (c) shows the radiation's spatial origin $L_{S_{(z, \alpha)}}$ along the z-axis, spectrally integrated. The two black lines indicate the wavelength $\lambda$ (b) or depth $z$ (c) of maximum radiation intensity $\mathrm{L}$ for a given coefficient of absorption $\alpha$. For fundamental analysis, the coefficient of absorption $\alpha$ in (b) and (c) is independent.

The absolute maximum of radiation intensity $L_{S_{(\lambda, \alpha)}}$ detectable at the upper surface $(\mathrm{y}=\mathrm{z}=0)$ is $9 \mathrm{pW} /\left(\mu \mathrm{m}^{2 *} \mathrm{sr}^{*} \mu \mathrm{m}\right)$ at $\alpha=1.31 / \mathrm{mm}$ and $6 \mu \mathrm{m}$ wavelength, marked with an $\mathrm{x}$ in figure 3 (b). The wavelength of maximum emission, indicated by the black line, slightly increases with the absorption coefficient. The radiation intensity's decrease at lower absorption coefficients is caused by marginal emissivity, at higher absorption coefficients it is evoked by excessive attenuation of radiation emitted within the specimen. Since the highest temperatures are in the joining zone around $z=1$, their radiation must travel a long way until reaching the surface. Thus, the radiation from the hot internal joining zone is significantly attenuated compared to colder ones closer to the surface, causing the shift of the radiation intensity's maximum to longer wavelengths with increasing coefficient of absorption (see equation 6).

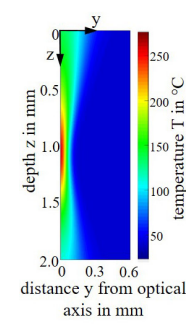

(a)

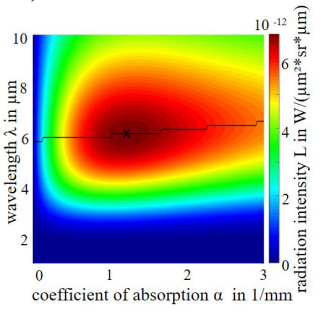

(b)

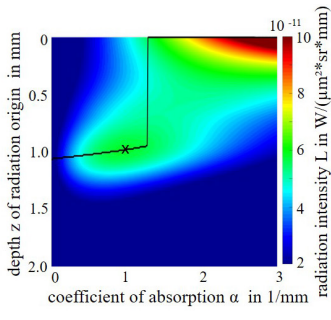

(c)
Fig. 3 (a) Temperature field of a tight seam without surface defects $25 \mu$ s after laser-material interaction (Material: COC, laser focus position $z_{S}=1.1 \mathrm{~mm}, z_{R}=0.3 \mathrm{~mm}, E=0.3 \mathrm{~J} / \mathrm{mm}, \alpha=0.281 / \mathrm{mm}$ ); Spectral (b) and spatial (c) composition of the radiation detectable at specimens surface in dependence on absorption. The two black lines indicate the wavelength $\lambda$ (b) or depth z (c) of maximum radiation intensity $\mathrm{L}$ for a given coefficient of absorption $\alpha$.

Analysing the depth of the radiation's origin in dependence on the coefficient of absorption in figure 3 (c), the maximum radiation $L_{S_{(z, \alpha)}}$ received from the joining zone $(z=1 \mathrm{~mm})$ is $58 \mathrm{pW} /\left(\mu \mathrm{m}^{2 *} \mathrm{sr}^{*} \mathrm{~mm}\right)$ at $\alpha=1.01 / \mathrm{mm}$. Up to a absorption coefficient of $\alpha=1.31 / \mathrm{mm}$ (see black line), most radiation $L_{S_{(z, \alpha)}}$ is emitted by the region around the joining zone. Above this value, the majority of the radiation is emitted by the surface. As already mentioned, attenuation of deeper regions is predominant with increasing absorption. Therefore, the radiation's origin shifts towards the surface with increasing coefficient of absorption.

In conclusion, the measurement wavelength should be around $5 \mu \mathrm{m}$ to $6 \mu \mathrm{m}$, since the emission maximum is located there for the process temperatures in laser transmission welding. The coefficient of absorption $\alpha$ should not be higher than approx. $11 / \mathrm{mm}$ to enable observation of the joining zone.

\subsection{Material Properties}

Figure 4 shows the coefficient of absorption for Topas 8007-04 and similar cyclic olefin copolymers required for material-specific signal analysis. Within the spectrum from $1.5 \mu \mathrm{m}$ to $2.5 \mu \mathrm{m}$, a Fourier transform infrared spectrometer extended with an integrating sphere was used. The equipment is described in Honnerova et. al [16]. Within the spectrum from $2.5 \mu \mathrm{m}$ to $3.2 \mu \mathrm{m}$ and $4.0 \mu \mathrm{m}$ to $6.5 \mu \mathrm{m}$, a Nicolet iS20 FTIR Spectrometer neglecting reflection was used. The values match the transmission data of other similar cyclic olefin copolymers provided by TOPAS Advanced Polymers for $0.2 \mu \mathrm{m}$ to $2 \mu \mathrm{m}$ wavelength, G-S Plastic Optics for $0.2 \mu \mathrm{m}$ to $3.2 \mu \mathrm{m}$ wavelength and Tydex LLC for $0.2 \mu \mathrm{m}$ to $13 \mu \mathrm{m}$ wavelength [17-19]. 


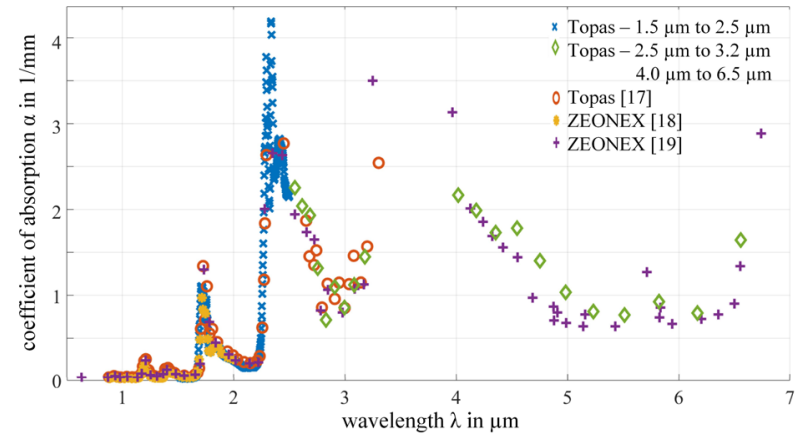

Fig. 4 Coefficient of absorption of cyclic olefin copolymers in dependence on wavelength [17-19]

Figure 5 shows the specific detectivity $D^{*}$ of five quantum-type infrared detectors at room temperature $(300 \mathrm{~K})$ available from Hamamatsu [20, 21], enabling costefficient integration without additional cooling. To evaluate the pyrometer chips, the signal characteristic must be checked in dependence on the weld seam position inside the material. In addition to a high signal, a maximum gradient along the seam position is required. Furthermore, a fast rise time is required for online process monitoring.

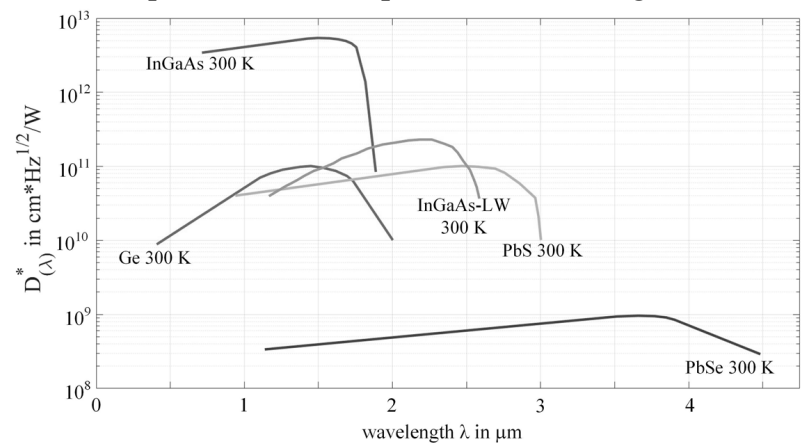

Fig. 5 Spectral response characteristics of different quantum-type infrared detectors [20,21]

\subsection{Weld Seam Monitoring}

Figure 6 (a) shows the computed radiation intensity $L_{S(\lambda)}$ at the specimen's surface for five different laser focus positions $z_{S}$, considering the material's coefficient of absorption $\alpha$ in dependence on wavelength given in figure 4 . The temperature fields are computed for $\mathrm{COC}$, using $0.3 \mathrm{~J} / \mathrm{mm}$ energy per unit length, a laser absorption coefficient $\alpha$ of $0.281 / \mathrm{mm}$ and a Rayleigh length $z_{R}$ of $0.3 \mathrm{~mm}$

The absolute maximum radiation intensity $L_{S(\lambda)}$ is $13 \mathrm{pW} /\left(\mu \mathrm{m}^{2 *} \mathrm{sr} * \mu \mathrm{m}\right)$ at a focal position $z_{S}$ of $0.8 \mathrm{~mm}$ and $5.7 \mu \mathrm{m}$ wavelength. The rise of the intensity at $1.7 \mu \mathrm{m}$ correlates with an increase of the materials' coefficient of absorption up to approx. $11 / \mathrm{mm}$ at the same wavelength. Overall with increasing wavelength up to $5.5 \mu \mathrm{m}$, the intensity drops between $3.2 \mu \mathrm{m}$ and $4 \mu \mathrm{m}$. Above $6.8 \mu \mathrm{m}$, the intensity decay is a result of the high coefficient of absorption of $\alpha=31 / \mathrm{mm}$ at these wavelengths. Above a wavelength of $6.5 \mu \mathrm{m}$, the intensity decreases according to Planck's law and high absorption. Shifting the laser focus downwards in the specimen (increasing $z_{S}$ ), reduces the radiation intensity at the surface. This is caused by decreased emission due to the lower temperatures and the simultaneously increased absorption, caused by the longer optical path length of emitted radiation until reaching the upper surface.

Figure 6 (b) shows the pyrometer signal in dependence on the laser focus position for five different detectors. The signal is calculated by multiplying the radiation intensity $L_{S(\lambda)}$ emitted at the surface (figure 6 (a)) with the specific detectivity $\mathrm{D}^{*}$ (figure 5) and subsequent spectral integration.

According to the previously calculated radiation intensity, the pyrometer signal decreases when shifting the laser focus downwards. Evaluating the infrared detectors, the maximum signal of $\left.0.15 \mathrm{~cm}^{*} \mathrm{~Hz}^{1 / 2}\right)^{*}\left(\mu \mathrm{m}^{2 *} \mathrm{sr}\right)$ is achieved for the $\mathrm{PbS}$, followed by the InGaAs-LW (0.09) and the standard InGaAs (0.06) detector. All three detectors are suitable for process monitoring. Detectors made of $\mathrm{PbSe}$ and Ge are not applicable because of the weak signal.

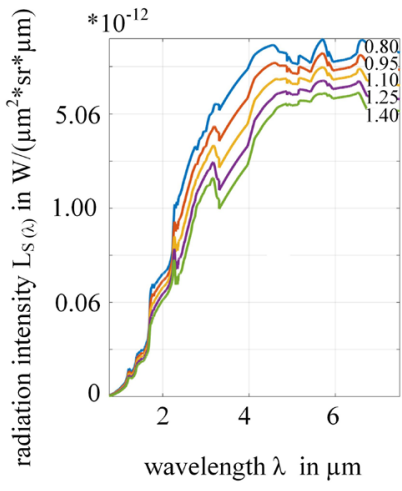

(a)

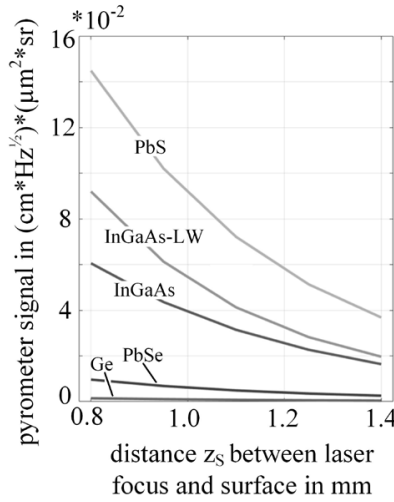

(b)
Fig. 6 (a) Spectral radiation intensity $L_{S(\lambda)}$ at upper surface in dependence on wavelength for different laser focus positions $\mathrm{zS}$ inside the specimen and (b) pyrometers' electric signals in dependence on laser focus position zs.

Considering speed and commercial availability, InGaAsdetectors are preferred for online process monitoring in laser transmission welding. Since only a filter blocking all wavelengths downwards the laser wavelength was available and its computed signal is higher, the long-wavelength type InGaAs-chip was chosen for experimental tests.

\section{Experimental Verification}

To verify the measurement spectrum recommended by the computations, a fixed-focus optic is set up. Welding tests of COC (Topas 8007-04) are performed and monitored using a pyrometer.

\subsection{Setup}

Figure 1 (a) shows the experimental setup used for welding. The fixed-focus setup consists of a breadboard, where a rail carrying the optical elements is mounted on slides. The beam delivered by a thulium fiber laser $\left(\lambda=1940 \mathrm{~nm}, \mathrm{M}^{2} \leq 1.2\right)$ is guided through an adjustable beam expander and a Galilean telescope with high NA ( $>0.6$ ) focusing lens. A fine threaded spindle moves the rail, enabling the variation of the distance between the optics and the specimen. Meanwhile the distances between the optics remain unchanged. A measurement system with $0.01 \mathrm{~mm}$ resolution controls the rail's position. A clamping device with a conical slit hole fixes the two specimens 
$\left(50 \times 50 \times 1 \mathrm{~mm}^{3}\right.$ each) in overlap. A 2-axis linear system moves the specimens with up to $300 \mathrm{~mm} / \mathrm{s}$.

For process monitoring, a customized pyrometer based on Micro-Epsilon CTM-3CF1-22 with an optical filter blocking the laser wavelength is used. Due to the filter, the sensitivity is diminished to the range of $2 \mu \mathrm{m}$ to $2.5 \mu \mathrm{m}$. The analog output is processed with $100 \mathrm{kHz}$ using a cRIO-9035. The diameter of the measurement spot is $1.5 \mathrm{~mm}$, where the spot size refers to $90 \%$ of the radiation energy. The distance from the front edge of the pyrometer's optic to the measurement spot is $36 \mathrm{~mm}$. Due to off-axis integration, the pyrometer is aligned with 25 degree inclination against the horizontal y-axis. This enlarges and distorts the spot, resulting in an elliptic spot with $3.1 \mathrm{~mm}$ in $\mathrm{x}$ and $5.6 \mathrm{~mm}$ in $\mathrm{y}$ direction, where $\mathrm{x}$ is the feed direction.

\subsection{Weld Seam Assessment}

At least seven welds per setting were processed at $60 \mathrm{~W}$ and a $200 \mathrm{~mm} / \mathrm{s}$ feed rate at five different laser focus positions $Z_{\text {rel }}$ between $0.3 \mathrm{~mm}$ and $0.7 \mathrm{~mm}$. The values of $z_{\text {rel }}$ represent the measured shift of the laser focus in air, not inside the specimen. At $z_{r e l}=0.55 \mathrm{~mm}$, the laser focus position is in the joining zone. After joining, approx. $50 \mu \mathrm{m}$ thick cuts were prepared in the middle of the specimen using a rotary microtome (Leica RM2255). The cuts were photographed in polarized light using a transmitted light microscope (Olympus BX53M). Afterwards, the weld seam area is framed and measured using the image processing softwares Fiji and OLYMPUS Stream Essentials Version 2.3.

Figure 7 (a) shows photographs of typical weld seams and figure 7 (b) shows the distance between the specimen's surface and the upper $(\mathrm{U})$ and lower $(\mathrm{L})$ end of the seam in dependence on the laser focus position $z_{\text {rel }}$. Figure 7 (c) shows the corresponding pyrometer signal. The signals were detected along a $20 \mathrm{~mm}$ long section the middle of the specimen. The seams are divided in four classes:

- Class 0:

The welds of class $0\left(z_{\text {rel }}=0.7 \mathrm{~mm}\right)$ are inadequate as no firm connection of the joining partners is given. The joining partners are loose after welding, even if the visible heat affected zone covers both partners. However, as mentioned at the end of chapter 2.1 , the temperature $T_{v i s}$ leading to a visible seam is less than the melt temperature $T_{\text {melt }}$ needed for bonding. The pyrometers signal is below threshold and only noise with a mean value of $0.8 \mathrm{mV}$ is detected.

- Class $\mathrm{I}_{\mathrm{A}, \mathrm{B}}$ :

Class I $\left(z_{\text {rel }}=0.6 \mathrm{~mm}\right.$ and $\left.0.5 \mathrm{~mm}\right)$ represents the desired result. Since line energy and focal position are well-matched, both joining partners are connected firmly and a sufficient distance $U(0.1 \mathrm{~mm}<U<0.2 \mathrm{~mm})$ between the seam and the surface is given. The pyrometer signals are $1.3 \mathrm{mV}$ and $1.5 \mathrm{mV}$, respectively.

- Class II:

In this marginal case $\left(z_{r e l}=0.4 \mathrm{~mm}\right)$, a firm connection is given but the surface may or may not show noticeable defects as the weld seam just reaches the surface $(U=0)$. The specimens have to be checked and a focus adjustment downwards is advised. The pyrometer signal is $2.6 \mathrm{mV}$.

- Class III:

Although both joining partners are connected tightly, the welds of class III $\left(z_{r e l}=0.3 \mathrm{~mm}\right)$ are inadequate since the surface is damaged by a palpable bulge caused by the weld seam. The pyrometer signal is $4.2 \mathrm{mV}$.

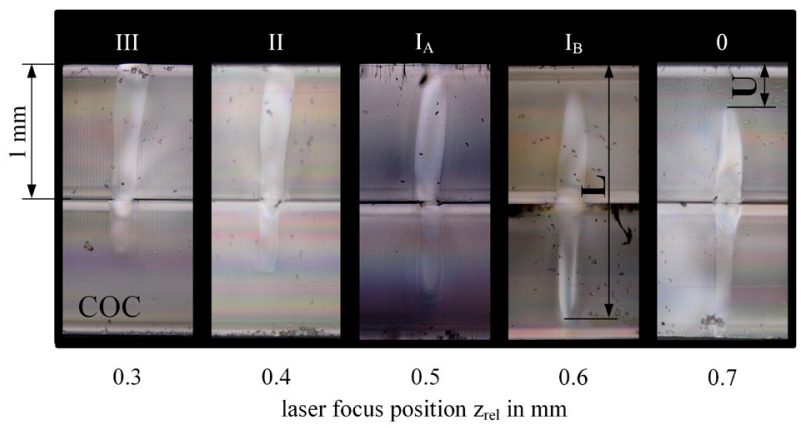

(a)

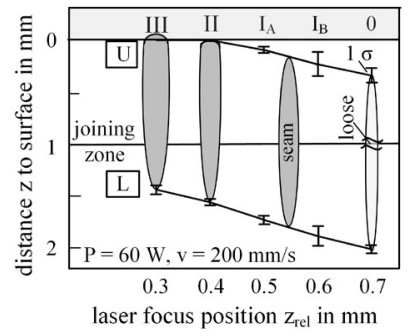

(b)

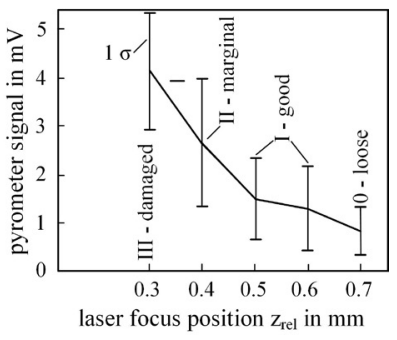

(c)
Fig. 7 (a) Photographs of typical weld states and (b) distance between specimen's surface and upper (U) and lower (L) end of seam in dependence on relative laser focus position $\mathrm{Z}_{\text {rel }}$. (c) Pyrometer signal for loose $(0)$, undamaged $\left(\mathrm{I}_{\mathrm{A}, \mathrm{B}}\right)$, marginal (II) and damaged (III) specimen averaged along $20 \mathrm{~mm}$ seam length. Material: Topas 8007-04. Laser wavelength $=1940 \mathrm{~nm}$, laser power $=60 \mathrm{~W}$, feed rate $=200 \mathrm{~mm} / \mathrm{s}$, Rayleigh length $=0.3 \mathrm{~mm}$.

The experiment shows that proper and faulty parts can be distinguished using a pyrometer with a measurement spectrum of $2 \mu \mathrm{m}$ to $2.5 \mu \mathrm{m}$. Both a molten surface and a faulty joint can be distinguished from proper results. Operating the process between $1.3 \mathrm{mV}$ and $1.5 \mathrm{mV}$ signal ensures the desired result of tight seams without surface defects. Additionally, weld seams processed with up to $2.6 \mathrm{mV}$ signal can be acceptable, since the surface may or may not show noticeable defects.

\section{Conclusion}

To fulfill the high demands on quality in medical industry, pyrometer based process monitoring is used. Interaction and impact of material and measurement parameters on the signal are analysed in simulations to preselect the measurement hardware. Welding tests using a fixed-focus optic prove that good seams can be distinguished from loose or damaged ones.

Since the measurement is performed during the process, closed loop control of focal position or other parameters is possible and scheduled as the next task. In addition, adapted intensity distributions for process stabilization and transformation of weld seam geometry will be investigated.

\section{Acknowledgements}

The authors gratefully thank the Bavarian Ministry for Economic Affairs, Media, Energy and Technology for funding the project "3D-Laspyrint-Scanner" and the project partners Bayerisches Laserzentrum $\mathrm{GmbH}$, Gerresheimer 
Regensburg GmbH, LPKF WeldingQuipment GmbH, Micro-Epsilon Messtechnik GmbH \& Co. KG and Nexlase $\mathrm{GmbH}$ for kindly providing technical support and good teamwork. Thanks to Futonics Laser $\mathrm{GmbH}$ for providing a laser.

\section{References}

[1] N. Nam-Phong, M. Brosda, A. Olowinsky and A. Gillner: J. Laser Micro/Nanoengin., 14, (2019) 255.

[2] S. Polster: "Laserdurchstrahlschweissen transparenter Polymerbauteile“ ed. by M. Geiger and K. Feldmann, (Meisenbach, Bamberg, 2009) p. 73.

[3] V. Mamuschkin, A. Haeusler, C. Engelmann, A. Olowinsky and H. Aehling: J. of Laser Appl., 29, (2017) 22409.

[4] V. Wippo et al.: Phys. Proc., 39, (2012) 128.

[5] A. Schmailzl, S. Steger and S. Hierl: Laser Tech. J., 12, (2015) 34

[6] A. Schmailzl, B. Quandt, M. Schmidt and S. Hierl: Proc. CIRP, 74, (2018) 524.

[7] A. Schmailzl et al.: Int. J. Heat Mass Transf., 146, (2020) p. 118814.

[8] R. Gardon: J. Am. Ceram. Soc., 39, (1956) 278.

[9] H. O. McMahon: J. Opt. Soc. Am., 40, (1950) 376.
[10] A. Schmailzl, S. Hüntelmann, T. Loose, J. Käsbauer, F. Maiwald and S. Hierl: Math. Mod. Weld Phenom., 13, (2019) 965.

[11]M. Geiger, T. Frick and M. Schmidt: Prod. Eng. - Res. and Dev., 3, (2009) 49.

[12]A. Schmailzl, B. Geißler, F. Maiwald, T. Laumer, M. Schmidt and S. Hierl: Lasers in Manuf. Conf., (2017).

[13]F. Maiwald, S. Englmaier and S. Hierl: Proc. CIRP, 94, (2020) p. 687.

[14] TOPAS Advanced Polymers: "Processing datasheet: TOPAS 8007S-04”, (Topas, Raunheim, 2019) p. 1.

[15]D. P. DeWitt and G. D. Nutter: "Theory and practice of radiation thermometry", (Wiley, New York, 1988).

[16] P. Honnerová, J. Martan, Z. Veselý and M. Honner: Sci. Rep., 7, (2017) p. 1386.

[17]TOPAS Advanced Polymers: "Topas COC: Cyclic olefin copolymer", (Topas, Raunheim, 2019) p. 10.

[18] G-S Plastic Optics: "Material Transmission Charts: E48R", (Online, Rochester, 2020).

[19] Tydex: "Thz Materials datasheet", (Tydex LLC, St. Petersburg, 2020) p. 3.

[20]Hamamatsu Photonics: "Technical Information SD-12", (Hamamatsu, Hamamatsu City, 2020) p. 8.

[21] Hamamatsu Photonics: "Infrared Detectors: Selection Guide”, (Hamamatsu, Hamamatsu City, 2011) p. 9.

(Received: August 13, 2020, Accepted: January 3, 2021) 